\title{
New guidelines for preoperative fasting
}

\section{Gastric physiology}

The basic physiology of digestion and gastric emptying has been known since before the discovery of general anaesthesia. In 1833, Beaumont ${ }^{1}$ published direct observations of a patient who suffered a gunshot wound which healed leaving a gastric fistula. He observed that solid foods, such as meat and potatoes, which were stored in the stomach and took 3-6 hr to be converted into semifluid chyme and to pass completely from the stomach. He also recorded that clear fluids are "not affected by gastric juice and are emptied almost as soon as they enter the stomach." Modern dual isotope studies, ${ }^{2}$ in which solids and liquids are tagged with different radioactive isotopes, confirm Beaumont's findings that solids require several hours for emptying while clear liquids take less than two hours.

The stomach is a very distensible storage organ which can accommodate more than one $\mathrm{L}$ during eating and drinking before there is any increase in intragastric pressure. ${ }^{3}$ Gastric secretions mix with food to break it down into small particles to form semi-fluid chyme which empties slowly through the pylorus into the small intestine. Most of the peristaltic contractions in the antrum are weak and mix the food and gastric secretions. Strong contractions occur about $20 \%$ of the time and their intensity determines the rate of stomach emptying. Increased volume in the stomach promotes emptying, not by increasing intragastric pressure, but by stretching of the stomach wall which elicits reflexes that increase the activity of the pyloric pump, while slightly inhibiting the tone of the pyloric sphincter. Certain foods, particularly meat, cause the release of gastrin from the antral mucosa; this stimulates secretion of highly acid gastric juice from the fundus and also stimulates the pyloric pump to promote gastric emptying.

The presence of excess chyme in the duodenum evokes nervous reflexes in the duodenal wall that inhibit gastric emptying. Factors that excite these enterogastric reflexes include distention of the duodenum, acidity and osmolality of the chyme, and the presence of certain breakdown products, especially from proteins and fats. ${ }^{3}$ The upper intestine also releases hormones, mainly in response to fats, that delay gastric emptying by inhibiting the pyloric pump and increasing the tone of the pyloric sphincter.

Indigestible solids that cannot be broken down to $<2$ mm diameter are emptied by a different mechanism during the fasting state. A distinct four-phase cycle, the interdigestive myoelectric complex, ${ }^{4}$ recurs approximately every two hours. During the second hour of the cycle the pylorus remains open and larger particles are emptied. This cycle explains why solids such as peas and carrots (with a high cellulose content which is broken down very slowly) often remain in the stomach for many hours after easily digestible solids have emptied.

Emptying of liquids depends on the pressure gradient between the stomach and the duodenum, the intragastric pressure being generated by slow, sustained gastric contractions in the proximal portion of the stomach. Tonic contraction of the pyloric sphincter, which prevents the passage of food particles $>2 \mathrm{~mm}$ diameter, allows water and other fluids to empty easily through the small opening in the sphincter. ${ }^{5}$ Emptying of neutral, iso-osmolar and calorically inert solutions is rapid. Half of a 500 $\mathrm{ml}$ bolus of isotonic saline is emptied in $12 \mathrm{~min}^{6}$ The finding of residual gastric fluid volume $>200 \mathrm{ml}$ at 30 min after ingestion of $750 \mathrm{ml}$ of normal saline indicates abnormal gastric retention. ${ }^{7}$ Hypertonic solutions and those which contain acid, fat, or certain amino acids produce a subsequent delay in gastric emptying when they pass into the small bowel and activate inhibitory reflexes. ${ }^{2}$

\section{Delayed gastric emptying}

Anatomical obstruction of the antro-pyloric junction and immediate post-duodenal bulb area result in gastric retention. Indigestible solids are the first to be retained, followed by digestible solids and finally liquids. ${ }^{2}$ Motility disorders of the antrum allow normal emptying of liquids but delay the emptying of solids, whereas motility disorders of the fundus may delay both solid and liquid emptying. Metabolic disturbances that may be associated with delayed gastric emptying include hyperglycaemia, hypokalaemia, hypercalcaemia, hypocalcaemia, hypomagnesemia, hypothyroidism, uraemia, and hepatic coma. Only hyperglycaemia has been adequately examined; a blood glucose level $>11.1 \mathrm{mmol} \cdot \mathrm{L}^{-1}$ in normal persons does not affect emptying of saline solution but delays emptying of liquid protein-fat meals. ${ }^{8}$

Gastroparesis is one of the gastrointestinal compli-

From the Foothills Hospital at the University of Calgary, Calgary, Alberta 
cations of long-standing, poorly controlled, insulindependent diabetes mellitus. ${ }^{9}$ However, delayed gastric emptying correlates poorly with symptoms of prolonged post-prandial epigastric fullness with intermittent nausea. Delayed emptying of solids is the most consistent abnormality, although, as the disease progresses, retention of liquids also occurs. Diabetic patients, including those with chronic nausea and vomiting, have been shown by some investigators to have normal gastric emptying of liquids and easily digestible solids, ${ }^{10,11}$ while others found delayed emptying of both liquids and solids in 50\% of diabetic patients. ${ }^{12}$ There are no reports of gastric fluid studies in diabetic patients undergoing surgical procedures.

The effect of vagotomy and pyloroplasty on gastric emptying is not permanent, returning to normal within three years. ${ }^{13}$ Gastro-oesophageal reflux is associated with considerable solid food retention in $57 \%$ of patients whereas the remainder have normal or rapid emptying. Neither gastro-oesophageal reflux nor gastric ulcer disease delay gastric emptying of liquids. ${ }^{14,15}$

In the absence of any of the diseases or conditions that delay gastric emptying, preoperative gastric emptying is normal unless the patient has received an opioid premedication. All opioids cause marked delay ${ }^{16}$ as do the belladonna group of drugs, but to a lesser extent. ${ }^{17}$ However, in healthy elective patients the administration of a morphine and atropine premedication given $1-2 \mathrm{hr}$ afier ingestion of oral fluid does not increase the residual gastric fluid volume at induction of anaesthesia, ${ }^{18,19}$ presumably because gastric emptying occurred before the onset of the pharmacological effect of the premedication. Metoclopramide, domperidone and cisapride increase the rate of gastric emptying; metoclopramide accelerates emptying of liquids but not solids, ${ }^{20}$ and the delay induced by morphine is only reversed by cisapride. ${ }^{21}$ In studying the effect of anxiety on gastric emptying in preoperative patients, it is important to differentiate anxiety state, which is a transitory emotional condition, from long-term anxiety trait, which is the tendency to perceive a wide variety of situations as threatening. Preoperative anxiety in elective surgical patients has been shown to have either no effect ${ }^{22}$ or to be delayed; ${ }^{23}$ the delay only occurred in patients who became apprehensive while awaiting surgery (high state) but who normally had a low predisposition to anxiety (low trait).

\section{Fasting guidelines and pulmonary aspiration}

Fasting guidelines in most textbooks published before the 1960's distinguished between liquids and solids, ${ }^{24,25}$ allowing clear fluids until two or three hours before elective surgery. Since that time most authors have applied "NPO after midnight" to liquids as well as to solids. ${ }^{26}$ While authors correctly state that pulmonary aspiration of gas- tric contents is a leading cause of anaesthetic-related mortality, they do not mention that virtually all fatal cases occur in emergency abdominal and obstetrical procedures in which there are complicating factors such as recent food and fluid intake, trauma, administration of narcotic analgesics, and difficult intubations. ${ }^{27}$ These factors do not normally apply to healthy, elective patients in whom the incidence of clinically important aspiration is less than $1: 10,000$ cases. ${ }^{27}$ In one prospective study in children, who are considered to be at higher risk, the incidence of clinically important aspiration was 1:40,000. ${ }^{28}$

For clinically important pulmonary damage to occur, the following factors must all be present:

1 Gastric contents must be of sufficient volume and acidity to cause pulmonary damage.

2 Intragastric pressure must be greater than resistance of the lower oesophageal sphincter.

3 Protective airway reflexes must be reduced or abolished.

Oral intake can only increase the risk of pulmonary damage if it increases the volume or acidity of gastric contents at induction of anaesthesia. It does not affect the tone of the lower oesophageal sphincter or the integrity of the protective airway reflexes. Ethical considerations prevent studies in humans to determine the volume and acidity required to cause pulmonary damage but Raido ${ }^{29}$ has recently shown that $>0.6 \mathrm{ml} \cdot \mathrm{kg}^{-1}$ was required in monkeys, equivalent in humans to $40 \mathrm{ml}$ injected into the trachea. The residual volume in the stomach required to produce aspiration of $40 \mathrm{ml}$ into the lungs was not estimated.

\section{Clinical studies}

In 1977 Hester and Heath ${ }^{30}$ recorded the volume and $\mathrm{pH}$ of gastric contents in patients who fasted from 4 to $24 \mathrm{hr}$. They found no correlation between duration of fast and either volume or $\mathrm{pH}$. Miller, Wishart and Nimmo ${ }^{18}$ subsequently studied two groups of healthy gynaecological patients who underwent elective surgery before 1030 a.m.; one group fasted from midnight, the other group had a light breakfast that consisted of one slice of buttered toast and one cup of tea or coffee 2-4 hr before surgery. There was no difference in residual gastric fluid volume of $\mathrm{pH}$ between the two groups and they therefore questioned whether even a four-hour fast was necessary. With this background, clinical studies were undertaken in adults at Foothills Hospital in Calgary to determine whether ingestion of oral fluid two to three $\mathrm{hr}$ before the scheduled time of surgery influenced the volume or $\mathrm{pH}$ of gastric fluid at the time of induction of anaesthesia. Our first study ${ }^{31}$ limited oral intake to $150 \mathrm{ml}$ water. There were no clinically important differences in either residual gastric fluid volume or $\mathrm{pH}$ be- 
TABLE I Gastric fluid volume: oral fluid versus NPO in adults

\begin{tabular}{|c|c|c|c|c|c|c|}
\hline \multirow[b]{2}{*}{ Year } & \multirow[b]{2}{*}{ Author } & \multirow[b]{2}{*}{ Oral intake } & \multicolumn{2}{|c|}{ Oral fluid } & \multicolumn{2}{|l|}{ NPO } \\
\hline & & & $\begin{array}{l}\text { Mean } \\
\text { fast }\end{array}$ & $\begin{array}{l}R G V^{*} \\
(m l)\end{array}$ & $\begin{array}{l}\text { Mean } \\
\text { fast }\end{array}$ & $\begin{array}{l}R G L^{*} \\
(m l)\end{array}$ \\
\hline 1983 & Miller et al. $(\mathrm{UK})^{18}$ & Toast and tea/coffee & $31 / 4 \mathrm{hr}$ & $11(0-43)$ & $9 \mathrm{hr}$ & $9(0-42)$ \\
\hline 1986 & Maltby et al. (Canada) ${ }^{31}$ & Water $150 \mathrm{ml}$ & $2 \frac{1}{2} \mathrm{hr}$ & $18(0-56)$ & $161 / 2 \mathrm{hr}$ & $27(0-80)$ \\
\hline 1987 & Sutherland et al. (Canada) ${ }^{35}$ & Water $150 \mathrm{ml}$ & $21 / 2 \mathrm{hr}$ & $21(0-50)$ & $131 / 2 \mathrm{hr}$ & $30(2-75)$ \\
\hline 1988 & Hutchinson et al. (Canada) ${ }^{32}$ & Coffee/juice $150 \mathrm{ml}$ & $2 \frac{1}{2} \mathrm{hr}$ & $24(0-96)$ & $141 / 2 \mathrm{hr}$ & $23(0-75)$ \\
\hline 1988 & McGrady et al. $(\mathrm{UK})^{36}$ & Water $100 \mathrm{ml}$ & $2 \mathrm{hr}$ & $17(4-52)$ & $12 \mathrm{hr}$ & $35(0-58)$ \\
\hline 1989 & Agarwal et al. (India) ${ }^{37}$ & Water $150 \mathrm{ml}$ & $2 \mathrm{l} / 2 \mathrm{hr}$ & $21(0-50)$ & $12 \mathrm{hr}$ & $30(0-75)$ \\
\hline 1989 & Scarr et al. (Canada) $)^{33}$ & Coffee/juice $150 \mathrm{ml}$ & $<3 \mathrm{hr}$ & $25(0-90)$ & $>8 \mathrm{hr}$ & $26(0-120)$ \\
\hline 1991 & Maltby et al. (Canada) ${ }^{34}$ & Coffee/juice, no limit & $<3 \mathrm{hr}$ & $22(3-70)$ & $>8 \mathrm{hr}$ & $25(0-107)$ \\
\hline 1991 & Ross et al. (USA) $)^{38}$ & Water $225 \mathrm{ml}$ & $1 / 2 \mathrm{hr}$ & $21 \pm 18$ & $>8 \mathrm{hr}$ & $30 \pm 22$ \\
\hline 1991 & Mahiou et al. (France) ${ }^{39}$ & PEG† $1000 \mathrm{ml}$ & $2 \mathrm{hr}$ & $38 \pm 18$ & $11 \mathrm{hr}$ & $35 \pm 15$ \\
\hline
\end{tabular}

Values are mean \pm SD (range).

$* \mathrm{RGV}=$ residual gastric volume. †PEG $=$ polyethylene glycol.

TABLE II Gastric fluid volume: oral fluid versus NPO in children

\begin{tabular}{|c|c|c|c|c|c|c|}
\hline \multirow[b]{2}{*}{ Year } & \multirow[b]{2}{*}{ Author } & \multirow[b]{2}{*}{ Oral intake $(\mathrm{ml})$} & \multicolumn{2}{|c|}{ Oral fluid } & \multicolumn{2}{|l|}{$N P O$} \\
\hline & & & $\begin{array}{l}\text { Mean } \\
\text { fast }\end{array}$ & $\begin{array}{l}R G V^{*} \\
\left.\mathrm{ml} \cdot \mathrm{kg}^{-1}\right)\end{array}$ & $\begin{array}{l}\text { Mean } \\
\text { fast }\end{array}$ & $\begin{array}{l}R G V^{*} \\
\left(\mathrm{ml} \cdot \mathrm{kg}^{-1}\right)\end{array}$ \\
\hline 1987 & Meakin et al. $(\mathrm{UK})^{40}$ & $\begin{array}{l}\text { Cereal }+ \text { milk } \\
\text { Orange juice } 10 \mathrm{ml} \cdot \mathrm{kg}^{-1}\end{array}$ & $\begin{array}{l}>5 \mathrm{hr} \\
2-4 \mathrm{hr}\end{array}$ & $0.39 \operatorname{SEM}(0.06)$ & $4 \mathrm{hr}$ & 0.25 (SEM 0.04) \\
\hline 1989 & Splinter el al. (Canada) ${ }^{41}$ & Apple juice $3 \mathrm{ml} \cdot \mathrm{kg}^{-1}$ & $21 / 4 \mathrm{hr}$ & $0.24(0.01-1.39)$ & $16 \mathrm{hr}$ & $0.43(0.01-1.65)$ \\
\hline 1989 & Sandhar et al. (Canada) ${ }^{42}$ & Apple juice $5 \mathrm{ml} \cdot \mathrm{kg}^{-1}$ & $21 / 2 \mathrm{hr}$ & $0.34(0.0-1.0)$ & $10 \mathrm{l} / 2 \mathrm{hr}$ & $0.25(0.0-1.1)$ \\
\hline 1989 & Schreiner et al. (USA) ${ }^{43}$ & Unlimited clear fluid & $21 / 2 \mathrm{hr}$ & $0.44 \pm 0.50$ & $131 / 2 \mathrm{hr}$ & $0.57 \pm 0.51$ \\
\hline
\end{tabular}

Values are mean $\pm \mathrm{SD}$ (range). ${ }^{*} \mathrm{RGV}=$ residual gastric volume.

tween those who drank and those who remained "NPO after midnight." Then we allowed $150 \mathrm{ml}$ coffee or fruit juice ${ }^{32}$ with similar findings.

In 1988 our fasting guidelines for ambulatory patients were changed to state that no solid food should be eaten on the day of surgery but that $150 \mathrm{ml}$ clear fluid (tea, coffee, apple juice, or water) could be taken until three hours before the scheduled time of surgery. Since gastric emptying of clear fluids is virtually complete within two hours, this allowed patients to have surgery one hour earlier if a change in the surgical schedule occurred.

In 1989, inpatient guidelines were changed to allow unrestricted clear fluid until three hours before surgery. Follow-up studies 33,34 on more than 400 patients were undertaken which confirmed that ingestion of fluid neither increased nor decreased gastric fluid volume or $\mathrm{pH}$ and the risk of pulmonary aspiration was therefore not affected. The majority of inpatients drank $100-400 \mathrm{ml}$, although one patient managed $1200 \mathrm{ml}$ and had $33 \mathrm{ml}$ residual gastric fluid volume. There was no correlation between the residual gastric volume or $\mathrm{pH}$ with either the volume ingested or the time from ingestion to sampling. Results of these and similar studies are summarized in Table I. Similar studies have been conducted in children with comparable results (Table II).

\section{Conclusion}

Editorials in anaesthetic journals ${ }^{44,45}$ have recommended that fasting guidelines should be revised. The routine order "NPO after midnight" for healthy elective patients should apply only to solids and clear liquids should be permitted on the day of operation. To allow for late changes in the surgical schedule and a minimum two hour emptying time, clear liquids should be allowed until three hours before the scheduled time of surgery or two hours before the actual time of surgery.

\section{References}

1 Beaumont $W$. Gastric Juice and the Physiology of Digestion. Plattsburgh: Allen 1833.

2 Minani H, McCallum RW. The physiology and pathology of gastric emptying in humans. Gastroenterology 1984; 86: 1592-610. 
3 Guyton AC. Textbook of Medical Physiology. 8th ed. Philadelphia: W.B. Saunders Company, 1991; 700-3.

4 Code $C F$, Marlett $J A$. The interdigestive myoelectric complex of the stomach and small bowel of dogs. J Physiol (Lond) 1975; 246: 298-309.

5 Meyer JH, Thomson JB, Cohen MB, et al. Sieving of solid food by the canine stomach and sieving after gastric surgery. Gastroenterology 1979; 76: 804-13.

6 Hunt JN. Some properties of an alimentary osmoreceptor mechanism. J Physiol (Lond) 1956; 132: 267-88.

7 Goldstein H, Boyle JD. The saline load test - a bedside evaluation of gastric retention. Gastroenterology 1965; 49 : 375-80.

8 MacGregor IL, Gueller R, Watts HD, Meyer JH. The effect of acute hyperglycemia on gastric emptying in man. Gastroenterology 1976; 70: 190-6.

9 Zitomer BR, Gramm HF, Kozak GP. Gastric neuropathy in diabetes mellitus: clinical and radiological observations. Metabolism 1968; 17: 199-211.

10 Loo FD, Palmer DW, Soergel KH, Kalbfleisch JH, Wood $C M$. Gastric emptying in patients with diabetes mellitus. Gastroenterology 1984; 86: 485-94.

11 Feldman M, Smith HJ, Simon TR. Gastric emptying of solid radiopaque markers: studies in healthy subjects and diabetic patients. Gastroenterology 1984; 87: 895-902.

12 Ziessman HA, Sahey FH, Collen MJ. Biphasic solid and liquid gastric emptying in normal controls and diabetics using continuous acquisition in $\mathrm{LAO}$ view. Dig Dis Sci 1992; 37: 744-50.

13 Cowley DJ, Vernon P, Jones T, Glass HI, Cox AG. Gastric emptying of solid meals after truncal vagotomy and pyloroplasty in human subjects. Gut 1972; 13: 176-81.

14 McCallum RW, Mensh $R$, Lange $R$. Definition of the gastric emptying abnormality present in gastroesophageal reflux patients. Gastroenterology 1981; 84: 741-6.

15 Miller LJ, Malagelada JR, Longstreth GF, et al. Dysfunctions of the stomach with gastric ulceration. Dig Dis Sci 1980; 25: 857-64.

16 Todd JG, Nimmo WS. Effect of premedication on drug absorption and gastric emptying. Br J Anaesth 1983; 55: 1189-93.

17 Davies JM, Davison JS, Nimmo WS, Hardy J-F, O'Sullivan $G$, Maltby $J R$. The stomach: factors of importance to the anaesthetist. Can J Anaesth 1990; 37: 896-906.

18 Miller M, Wishart HY, Nimmo WS. Gastric contents at induction of anaesthesia. $\mathrm{Br} \mathrm{J}$ Anaesth 1983; 55: 1185-8.

19 Maltby JR, Koehli N, Ewen A, Shaffer EA. Gastric fluid volume, $\mathrm{pH}$, and emptying in elective patients. Influences of narcotic-atropine premedication, oral fluid, and ranitidine. Can J Anaesth 1988; 35: 562-6.

20 Hancock BD, Brown-Jones E, Dixon R, Dymock IW, Cowley DJ. The effect of metoclopramide on gastric emptying of solid meals. Gut 1974; 15: 462-7.
21 Rowbotham DJ, Nimmo WS. Effect of cisapride on morphine-induced delay in gastric emptying. $\mathrm{Br} \mathbf{J}$ Anaesth 1987; 59: 536-9.

22 Marsh $R K H$, Spencer R, Nimmo WS. Gastric emptying and drug absorption before and after surgery. $\mathrm{Br} \mathbf{J}$ Anaesth 1983; 55: 242P.

23 Simpson $K G$, Stakes $A F$. Effect of anxiety on gastric emptying in preoperative patients. Br J Anaesth 1987; 59: $540-4$.

24 Buxton DW. Anaesthetics: Their Uses and Administration. London: Lewis 1920; 24-5.

25 Leigh MD, Belton MK. Pediatric Anesthesia. 2nd ed. New York: The Macmillan Company 1960; 274.

26 Cohen DD, Dillon GB. Anesthesia for Outpatient Surgery. Springfield, Illinois: CC Thomas 1970; 11.

27 Olsson GL, Hallen B, Hambraeus-Jonzon K. Aspiration during anaesthesia: a computer-aided study of 185,358 anaesthetics. Acta Anaesthesiol Scand 1986; 30: 84-92.

28 Tiret L, Nwoche Y, Hatton F, Desmonts JM, Vour'h $G$. Complications related to anaesthesia in infants and children: a prospective survey of 40,240 anaesthetics. Br J Anaesth 1988; 61: 263-9.

29 Raidoo DM, Rocke DA, Brock-Uine JG, Marszaler A, Engelrecht $H E$. Critical volume for pulmonary acid aspiration: reappraisal in a primate model. $\mathrm{Br} \mathrm{J}$ Anaesth 1990; 65: 248-50.

30 Hester JB, Heath $M L$. Pulmonary acid aspiration syndrome: should prophylaxis be routine? $\mathrm{Br} J$ Anaesth 1977; 49: 595-9.

31 Maltby JR, Sutherland AD, Sale JP, Shaffer EA. Preoperative oral fluids: is a five-hour fast justified prior to elective surgery? Anesth Analg 1986; 65: 1112-6.

32. Hutchinson A, Maltby $M R$, Reid CRG. Gastric fluid volume and $\mathrm{pH}$ in elective patients. Part I; Coffee or orange juice versus overnight fast. Can J Anaesth 1988; 35: 12-5.

33 Scarr M, Maltby JR, Jani K, Sutherland LR. Volume and acidity of residual gastric fluid after oral fluid ingestion before elective ambulatory surgery. Can Med Assoc J 1989; 141: 1151-4.

34 Maltby JR, Lewis P, Martin A, Sutherland LR. Gastric fluid volume and $\mathrm{pH}$ in elective patients following unrestricted oral fluid until three hours before surgery. Can J Anaesth 1991; 38: 425-9.

35 Sutherland AD, Maltby JR, Sale JP, Reid CRG. The effect of preoperative oral fluid and ranitidine on gastric fluid volume and pH. Can J Anaesth1987; 34: 117-21.

36 McGrady EM, MacDonald $A G$. Effect of the preoperative administration of water on gastric volume and $\mathrm{pH} . \mathrm{Br} J$ Anaesth 1988; 60: 803-5.

37 Agarwal A, Chari P, Singh H. Fluid deprivation before operation. Anaesthesia 1989; 44: 632-4.

38 Ross WT, Momany GM. Ranitidine (IV) and water (PO) 
increase gastric $\mathrm{pH}$ and decrease gastric residual volume in outpatients. Anesthesiology 1991; 75: A1110.

39 Mahiou P, Narchi P, Gory G, Veyrac P, Germond M, Bazin $G$. Is coloscopic preparation with oral polyethylene glycol 2 hours before general anesthesia safe in ambulatory patients? Anesthesiology 1991; 75: A1 108.

40 Meakin G, Dingwall $A E$, Addison GM. Effects of fasting and oral premedication on the $\mathrm{pH}$ and volume of gastric aspirate in children. Br J Anaesth 1987; 59: 678-82.

41 Splinter WM, Stewart JA, Muir JG. The effect of preoperative apple juice on gastric contents, thirst and hunger in children. Can J Anaesth 1989; 36: 55-8.

42 Sandhar BK, Goresky GV, Maltby JR, Shaffer EA. Effect of oral liquids and ranitidine on gastric fluid volume and $\mathrm{pH}$ in children undergoing outpatient surgery. Anesthesiology 1989; 71: 327-30.

43 Schreiner MS, Triebwassen A, Keon TP. Ingestion of liquids compared with preoperative fasting in pediatric outpatients. Anesthesiology 1990; 72: 593-7.

44 Coté CJ. NPO after midnight in children - a reappraisal. Anesthesiology 1990; 72: 589-92.

45 Goresky GV, Maltby JR. Fasting guidelines for elective surgical patients. Can J Anaesth 1990; 37: 493-5. 


\title{
Nouvelles recom- mandations sur le jeûne préopératoire
}

\author{
J. Roger Maltby MB FFARCS FRCPC
}

\section{La physiologie gastrique}

On connaissait la physiologie de la digestion et de la vidange gastrique avant la découverte de l'anesthésie. En 1833, Beaumont ' publie ses observations directes d'un patient blessé par un projectile d'arme à feu qui guérit tout en laissant une fistule gastrique. Il observe que la nourriture solide, comme la viande et les pommes de terre gardées en storage dans l'estomac se convertissent en 3 à 6 heures en chyme semi-fluide avant de quitter l'estomac. Il remarque aussi que les liquides clairs ne sont " pas affectés par le jus gastrique et se vident dès qu'ils atteignent l'estomac $»$. Des études isotopiques ${ }^{2}$ au cours desquelles les solides et les liquides sont marqués avec différents isotopes radioactifs, confirment les constatations de Beaumont selon lesquelles les solides prennent plusieurs heures pour s'évacuer alors que les liquides clairs prennent moins de deux heures.

L'estomac est un organe de storage dilatable qui peut recevoir plus d'un litre de solide et de liquide sans augmentation de la pression intragastrique. ${ }^{3}$ Les sécrétions gastriques se mêlent à la nourriture pour la broyer en petites particules qui forment un chyme semi-fluide qui se vide lentement dans le petit intestin par le pylore. En général, les contractions péristaltiques antrales sont faibles et mélangent la nourriture aux sécrétions gastriques. De fortes contractions surviennent $20 \%$ du temps et leur intensité détermine la vitesse de vidange de l'estomac. L'augmentation du volume intragastrique favorise l'évacuation non pas par élévation de la pression intragastrique mais bien par étirement des parois de l'estomac. Cet étirement a pour effet de provoquer les réflexes qui accroissent l'activité de la pompe pylorique, tout en inhibant légèrement le tonus du sphincter pylorique. Certaines nourritures, en particulier la viande, favorise la libération de gastrine par la muqueuse antrale; ce qui stimule la sécrétion des jus gastriques très acides du fundus en même temps que la pompe pylorique dans son travail de vidange.

La présence d'un excès de chyme dans le duodénum provoque des réflexes neuraux dans la paroi duodénale qui inhibent la vidange gastrique. Les facteurs qui excitent ces réflexes entérogastriques comprennent la distension duodénale, l'acidité et l'osmolalité du chyme et la présence de certains produits de dégradation comme les protéines et les graisses. ${ }^{3}$ Lintestin supérieur librère aussi des hormones, principalement en réponse aux graisses, qui retarde la vidange gastrique par inhibition de la pompe pylorique et augmentation du tonus du sphincter pylorique.

Les solides indigestes qui ne peuvent être brisées en particules de moins de $2 \mathrm{~mm}$ de diamètre sont évacués par un mécanisme différent pendant le jeûne. Un cycle distinct à quatre phases, le complexe interdigestif myoélectrique, ${ }^{4}$ recommence approximativement aux deux heures. Pendant la deuxième heure du cycle, le pylore demeure ouvert et les plus grosses particules se vident. $\mathrm{Ce}$ cycle explique pourquoi les solides comme les pois et carottes (avec un contenu élevé de cellulose qui est dégradée très lentement) demeurent plusieurs heures dans l'estomac après le départ des solides faciles à digérer.

La vidange des liquides dépend du gradient de pression entre l'estomac et le duodénum. La pression intragastrique est générée par les contractions lentes et soutenues de la portion proximale de l'estomac. La contraction tonique du sphincter pylorique, qui prévient le passage des particule de plus de $2 \mathrm{~mm}$ de diamètre, laisse passer l'eau et les autres liquides par une petite ouverture du sphincter. ${ }^{5}$ La vidange des solutions neutres, iso-osmolaires et non-calorifiques est rapide. La moitié d'un bolus de 500 $\mathrm{ml}$ de soluté physiologique se vide en douze minutes. ${ }^{6}$ Le fait de trouver un résidu gastrique plus grand que $200 \mathrm{ml} 30$ minutes après lingestion de $750 \mathrm{ml}$ de soluté physiologique indique une rétention gastrique anormale. ${ }^{7}$ Les solutions hypertoniques et celles qui contiennent des graisses, des acides ou certains acides aminés retardent l'évacuation quand ils passent dans le petit intestin par activation de réflexes inhibiteurs. ${ }^{2}$

\section{Le retard de la vidange gastrique}

L'obstruction anatomique de la jonction antro-pylorique et de la région duodénale post-bulbaire immédiate cause de la rétention gastrique. Les solides indigestes sont les premiers retenus, suivi des solides digestibles et finalement des liquides. ${ }^{2}$ La dyskinésie antrale permet la vidange normale des liquides mais retarde la vidange des solides. Par contre, la dyskinésie fundique peut retarder la vidange des liquides et des solides. Les perturbations métaboliques associées au retard de la vidange gastrique comprennent 
l'hyperglycémie, l'hypokaliémie, l'hypercalcémie, l'hypocalcémie, l'hypomagnésémie, l'hypothyroidie, l'urémie et le coma hépatique. Une seule de ces conditions a été étudiée à fond, l'hyperglycémie; une glycémie plus élevée que $11,1 \mathrm{mmol} \cdot \mathrm{L}^{-1}$ chez la personne normale n'affecte pas la vidange du soluté physiologique mais retarde la vidange des repas liquides de protéines et de graisses.

La gastroparésie représente une des complications gastro-intestinales du diabète établi mal contrôlé insulinodépendant. ${ }^{9}$ Cependant, la corrélation entre le retard de la vidange gastrique les sensations de plénitude épigastrique postprandiale avec nausées intermittentes du diabétique n'est pas évidente. Le retard de la vidange gastrique des solides est l'anomalie la plus constante bien qu'avec la progression de la maladie, la rétention des liquides finit aussi par survenir. Certains ont trouvé chez les diabétiques, incluant ceux qui présentent de la nausée et des vomissements chroniques, une durée de vidange gastrique normale pour les liquides et les solides facilement digestibles; ${ }^{10,11}$ d'autres, par contre, ont trouvé un retard à la vidange des liquides et des solides chez 50\% des diabétiques. ${ }^{12}$ On ne connaît pas d'études sur les liquides gastriques des diabétiques lors d'interventions chirurgicales.

Les effets de la vagotomie et de la pyloroplastie sur l'évacuation gastrique ne sont pas permanents. On peut s'attendre à un retour à la normale dans les trois ans. ${ }^{13}$ Le reflux gastro-oesophagien est associé à une rétention significative de la nourriture solide dans $57 \%$ des patients alors que les autres ont une vidange normale ou accélérée. Le reflux gastro-oesophagien et l'ulcère gastrique ne retardent pas la vidange des liquides. ${ }^{14,15}$

En l'absence de toute maladie ou condition qui pourrait la retarder la vidange gastrique, préopératoire est normale, à moins que le patient n'ait reçu une prémédication opiacée. Tous les opiacés causent un retard important ${ }^{16}$ ainsi qu'à un degré moindre les dérivés de la belladone. ${ }^{17}$ Cependant, chez les patients en bonne santé et programmés pour une chirurgie réglée, l'administration d'une prémédication de morphine et d'atropine 1-2 heures après lingestion de liquide par la bouche n'augmente pas le volume résiduel gastrique au moment de l'induction de l'anesthésie; ${ }^{18,19}$ on peut présumer que c'est parce que la vidange s'est produite avant le début des effets de la prémédication. La métoclopramide, le dompéridone et la cisapride augmentent la vitesse de l'évacuation gastrique; la métoclopramide accélère l'évacuation des liquides et non des solides, ${ }^{20}$ et le retard initié par la morphine n'est renversé que par la cisapride. ${ }^{21}$ En étudiant les effets de l'anxiété sur la vidange gastrique à la période préopératoire, il est important de distinguer l'état d'anxiété, qui est une condition émotionnelle transitoire, du trait anxieux qui consiste en une tendance à percevoir une grande variété de situations comme menaçantes. L'anxiété préopératoire rencontrée chez le patient en chirurgie réglée n'a pas d'effet démontrés ${ }^{22}$ ou retarde la vidange. ${ }^{23}$ Le retard survient seulement chez les patients en attente de chirurgie devenus craintifs mais qui normalement auraient une faible propension pour l'anxiété.

\section{Les recommandations sur le jeûne et l'aspiration pulmonaire}

Les lignes de conduite concernant le jeûne publiées dans la plupart des manuels avant 1960 distinguaient entre liquides et solides, ${ }^{24,25}$ et permettaient l'ingestion de liquides clairs jusqu'à 2 ou 3 heures avant la chirurgie réglée. Depuis ce temps, certains auteurs ont appliqué la règle du " jeûne absolu après minuit " que ce soit pour les liquides ou pour les solides. ${ }^{26}$ Alors que certains auteurs déclarent à juste titre que l'aspiration du contenu gastrique est la cause principale de mortalité reliée à l'anesthésie, ils ne prennent pas le peine de mentionner que pratiquement tous les cas de décès surviennent pendant les interventions abdominales ou obstétricales urgentes alors que les patients présentent des facteurs de complications comme l'ingestion récente de nourriture et de liquide, un traumatisme, l'administration d'opiacés et l'intubation difficile. ${ }^{27}$ Ces facteurs ne se rencontrent pas normalement dans une population de patients non tarés et en chirurgie réglée. L'incidence d'aspiration cliniquement décelable est dans ces cas de $1: 10,000 .{ }^{27}$ Lors d'une étude réalisée chez des enfants, qu'on considère à plus haut risque, lincidence d'aspiration cliniquement décelable n'est que de $1: 40,000 .{ }^{28}$

Pour qu'une atteinte pulmonaire clinique significative puisse survenir, tous les facteurs suivants doivent être présents:

1 Le contenu gastrique doit être en quantité et d'acidité suffisantes pour causer une atteinte pulmonaire.

2 La pression intragastrique doit être plus élevée que celle du sphincter inférieur de l'oesophage.

3 Les reflexes protecteurs des voies respiratoires doivent être diminués ou abolis.

L'ingestion peut augmenter le risque de dommages pulmonaires si elle augmente le volume ou l'acidité du contenu gastrique à linduction de l'anesthésie. Elle n'affecte pas le tonus du sphincter inférieur de l'oesophage ou l'intégrité des réflexes protecteurs. Des considérations éthiques empêchent de déterminer chez l'humain le volume et l'acidité requises pour causer des dommages aux poumons. Raidoo ${ }^{29}$ a toutefois montré récemment que moins de $0,6 \mathrm{ml} \cdot \mathrm{kg}^{-1}$ était nécessaire chez le singe, l'équivalent de $40 \mathrm{ml}$ injectés dans la trachée de l'humain. Le volume résiduel stomachal requis pour produire une aspiration de $40 \mathrm{ml}$ dans le poumon humain n'a pas été déterminé. 
TABLEAU I Le volume du contenu gastrique: liquides oraux vs jeûne absolu chez l'adulte

\begin{tabular}{|c|c|c|c|c|c|c|}
\hline \multirow[b]{2}{*}{ Année } & \multirow[b]{2}{*}{ Auteurs } & \multirow[b]{2}{*}{ Ingesta } & \multicolumn{2}{|l|}{ Ingesta } & \multicolumn{2}{|c|}{ Jeûne absolu } \\
\hline & & & $\begin{array}{l}\text { Jeûne } \\
\text { moyen }\end{array}$ & $V G R^{*}$ & $\begin{array}{l}\text { Jeûne } \\
\text { moyen }\end{array}$ & $V G R^{*}$ \\
\hline 1983 & Miller et al. (UK) ${ }^{18}$ & toast et thé/café & $31 / 4$ hres & $11(0-43)$ & 9 hres & $9(0-42)$ \\
\hline 1986 & Maltby et al. (Canada) $)^{35}$ & eau $150 \mathrm{ml}$ & $21 / 2$ hres & $18(0-56)$ & $161 / 2$ hres & $27(0-80)$ \\
\hline 1987 & Sutherland et al. (Canada) ${ }^{32}$ & eau $150 \mathrm{ml}$ & $21 / 2$ hres & $21(0-50)$ & $131 / 2$ hres & $30(2-75)$ \\
\hline 1988 & Hutchison et al. (Canada) ${ }^{32}$ & café/jus $150 \mathrm{ml}$ & $21 / 2$ hres & $24(0-96)$ & $141 / 2$ hres & $23(0-75)$ \\
\hline 1988 & McGrady et al. (UK) & eau $150 \mathrm{ml}$ & 2 hres & $17(4-52)$ & 12 hres & $35(0-58)$ \\
\hline 1989 & Agarwal et al. (Indes) $)^{37}$ & eau $150 \mathrm{ml}$ & $21 / 2$ hres & $21(0-50)$ & 12 hres & $30(0-75)$ \\
\hline 1989 & Scarret al. (Canada) ${ }^{33}$ & café/jus 150 & $>3$ hres & $25(0-90)$ & $>8$ hres & $26(0-120)$ \\
\hline 1991 & Maltby et al. (Canada) ${ }^{34}$ & café/jus, sans restriction & $>3$ hres & $22(3-70)$ & $>8$ hres & $25(0-107)$ \\
\hline 1991 & Ross et al. (USA) ${ }^{38}$ & eau 225 & $1 / 2$ hres & $21 \pm 18$ & $>8$ hres & $30 \pm 22$ \\
\hline 1991 & Mahiou et al. (France) ${ }^{39}$ & PEG $† 1000 \mathrm{ml}$ & 2 hres & $38 \pm 18$ & 11 hres & $35 \pm 15$ \\
\hline
\end{tabular}

Valeurs $=$ moyennes \pm écart type (étendue).

*VGR $=$ volume gastrique résiduel. $\uparrow \mathrm{PEG}=$ polyéthylène glycol .

TABLEAU II Volume du contenu gastrique: ingestion vs jeûne absolu

\begin{tabular}{|c|c|c|c|c|c|c|}
\hline \multirow{2}{*}{\multicolumn{2}{|c|}{ Année Auteurs }} & \multirow[b]{2}{*}{$\begin{array}{l}\text { Ingesta } \\
(m l)\end{array}$} & \multicolumn{2}{|l|}{ Ingestion } & \multicolumn{2}{|c|}{ Jet̂ne absolu } \\
\hline & & & $\begin{array}{l}\text { Jeûne } \\
\text { moyen }\end{array}$ & $\begin{array}{l}V G R \\
\left(m l \cdot k g^{-1}\right)\end{array}$ & $\begin{array}{l}\text { Jeûne } \\
\text { moyen }\end{array}$ & $\begin{array}{l}V G R \\
\left(m l \cdot k g^{-1}\right)\end{array}$ \\
\hline 1987 & Meakin et al. (UK) & $\begin{array}{l}\text { céréales et lait jus d'orange } 10 \\
\mathrm{ml} \cdot \mathrm{kg}^{-1}\end{array}$ & $\begin{array}{l}>5 \text { hres } \\
2-4 \text { hres }\end{array}$ & 0,39 (SEM 0,06) & 4 hres & 0,25 (SEM 0,04) \\
\hline 1989 & Splinter et al. (Canada) ${ }^{41}$ & jus de pomme $3 \mathrm{ml} \cdot \mathrm{kg}^{-1}$ & $21 / 4$ hres & $0,24(0,01-1,39)$ & 16 hres & $0,43(0,01-1,65)$ \\
\hline 1989 & Sandhar et al. (Canada) ${ }^{42}$ & jus de pomme $5 \mathrm{ml} \cdot \mathrm{kg}^{-1}$ & $2 \frac{1}{2} / 2$ hres & $0,34(0,0-0,1)$ & $101 / 2$ hres & $0,25(0,01-1,1)$ \\
\hline 1990 & Schreiner $e l$ al. $(\mathrm{USA})^{43}$ & liquides clairs sans restrictions & $21 / 2$ hres & $0,44 \pm 0,50$ & $13 \% / 2$ hres & $0,57 \pm 0,51$ \\
\hline
\end{tabular}

Valeurs $=$ moyennes \pm écart type (étendue).

*VGR = volume gastrique résiduel.

\section{Etudes cliniques}

En 1977, Hester et Heath ${ }^{30}$ ont mesuré le volume et le $\mathrm{pH}$ du contenu gastrique chez des patients à jeun depuis 4 à 24 heures. Il n'ont établir de corrélation entre la durée du jeûne et le volume ou le $\mathrm{pH}$. Miller et al. ${ }^{18}$ ont étudié par la suite deux groupes de patientes gynécologiques non tarées qui subissaient une chirurgie réglée avant 10 heures 30: un groupe jeûnait depuis minuit; l'autre avait eu droit à un léger déjeuner consistant en une toast beurrée et une tasse de thé ou de café 2-4 heures avant la chirurgie. Il n'ont pas trouvé de différence de volume gastrique résiduel ou de $\mathrm{pH}$ entre les deux groupes et ils se sont demandés si un jeûne de quatre heures en valait la peine. Avec ces données comme base, des études cliniques ont été entreprises chez des adultes au Foothills Hospital de Calgary pour déterminer si lingestion de liquide $2-3$ heures avant l'heure programmée pour la chirurgie influençait le volume ou le $\mathrm{pH}$ du contenu gas- trique au moment de l'induction de l'anesthésie. Notre première étude ${ }^{31}$ limitait l'ingestion de liquide à $150 \mathrm{ml}$ d'eau. Nous n'avons pas trouvé de différence significative de volume résiduel ou de $\mathrm{pH}$ entre ceux qui avaient bu et ceux qu'on gardait au jeûne absolu depuis minuit. Ensuite, on a permis $150 \mathrm{ml}$ de café ou de jus de fruit avec les mêmes résultats. ${ }^{32}$

En 1988, nos lignes de conduite sur le jeûne furent changées pour spécifier que la nourriture solide demeurait interdite le jour de la chirurgie mais que $150 \mathrm{ml}$ de liquide clair (thé, café, jus de pomme ou eau) pouvaient être ingérés jusqu'à trois heures avant la chirurgie. Comme l'évacuation gastrique des liquides clairs est pratiquement complète après deux heures, ceci permettait de procéder à l'intervention une heure plus tôt que prévu si un changement à l'horaire du programme opératoire l'exigeait.

En 1989, les recommandations pour les patients hospitalisés furent encore changées pour enlever toute res- 
triction sur l'ingestion des liquides clairs jusquà trois heures avant la chirurgie. Un suivi ${ }^{33,34}$ de plus de 400 patients fut entrepris. Il confirma que l'ingestion de liquide n'augmentait, ni ne diminuait le volume et le $\mathrm{pH}$ gastriques. La majorité des patients ingéra de 100 à $400 \mathrm{ml}$ de liquide. Un patient réussit à lui seul à en ingérer 1200 $\mathrm{ml}$ et n'avait que $33 \mathrm{ml}$ de volume résiduel. Nous n'avons pas trouvé de corrélation entre le volume résiduel ou le $\mathrm{pH}$ gastrique et le volume ingéré ou le moment de l'ingestion. Les résultats de cette étude et des autres études identiques sont résumés au Tableau I. Des études similaires ont été menées chez les enfants avec les mêmes résultats (Tableau II).

\section{Conclusion}

Les éditoriaux des revues d'anesthésie ${ }^{44,45}$ réclamaient une révision des lignes de conduite concernant le jeûne. L'ordonnance de jeûne après minuit pour des patients non tarés programmés pour une chirurgie réglée ne devrait s'appliquer que pour les solides: les liquides clairs devraient être permis le jour de l'opération. Pour permettre des changements de dernière minute au programme opératoire et un minimum de deux heures pour l'évacuation gastrique, des liquides clairs devraient être permis jusqu'à trois heures avant l'heure proposée pour la chirurgie et jusqu'à deux heures avant l'heure réelle de la chirurgie.

\section{Références}

(Voir page R115) 